\title{
A Localization Algorithm Based on Particle Swarm Optimization and Quasi-Newton Algorithm for Wireless Sensor Networks
}

\author{
Jingang Cao \\ Department of Computer, North China Electric Power University, Baoding 071003, China
}

\begin{abstract}
The position information is becoming more and more important for application in WSNs. So aiming at the location problem, the paper proposes a localization algorithm based on PSO (particle swarm optimization) and Quasi-Newton algorithm, which use PSO to get a satisfying value and then use it as the initial value of Quasi-Newton algorithm to iterate. The simulation experiments are carried out. The results show that the proposed algorithm not only fastens the localization speed and has improved the localization precision effectively.
\end{abstract}

Key words: PSO, Quasi-Newton algorithm, WSN, localization accuracy.

\section{Introduction}

WSNs (wireless sensor networks) are composed of hundreds of sensor nodes which are distributed in a wide area. These nodes communicate with each other either directly or through other nodes and WSNs can obtain relevant environmental data. So WSNs can be used in military, health care, smart home, environmental monitoring, emergency rescue and disaster relief, etc. [1, 2]. Most applications of WSNs need know the location of nodes beforehand so as to adjust the route, manage network and track target, etc. As far as the collected data is concerned, the data will lose its proper value if it does not know the location information. Therefore, the node localization technology is a key technology for WSNs and also it is an important research topic in WSNs [3].

According to localization mechanism of WSNs, the node localization methods can be divided into range-based and range-free approaches [2]. Compared with range-free approach, range-based approach can obtain higher localization accuracy. Such localization

Corresponding author: Jingang Cao, lecturer, research fields: wireless sensor networks, artificial intelligence. E-mail: caojg168@126.com. algorithm include RSSI (received signal strength indicator), TOA (time of arrival), TDOA (time difference of arrival), and AOA (angle of arrival), etc.

Trilateral positioning method, triangulation method and Least squares estimation method are three common localization methods [4]. But all of them have some disadvantages. As for trilateral localization method, the localization accuracy is not satisfactory when there are ranging errors; The problem of triangulation is that even if only minor changes of the external environment can lead to bad ranging results; Least Squares Estimation method can improve the localization accuracy, but it is easy to be affected by environment and maybe have big ranging errors, so the localization accuracy is not satisfactory.

In order to reduce the effects of ranging errors on localization accuracy, PSO (particle swarm optimization) was used to optimize the localization results, which improve the localization accuracy to some extent, but when PSO algorithm evolves into the later stage, the particle is easy to fall into the local minimum area, and it is difficult to converge to the better results. Aiming at this problem, the paper presents a hybrid algorithm based on particle swarm 
optimization algorithm and Quasi-Newton algorithm for WSNs node localization in order to improve localization accuracy.

\section{Ranging Method of RSSI}

In the ranging phase, the most important problem is how to calculate the distance between unknown nodes and anchor nodes to improve the location precision. There are many researches about how to eliminate channel interference and reduce RSSI ranging errors $[5,6]$. There are some common wireless signal propagation models: free-space propagation model, logarithmic distance path loss model and Hata model, etc.

In free space, according to Friis formula, the received signal power of the receivers $P_{r}(d)$ is

$$
P_{r}(d)=\frac{P_{t} G_{t} G_{r} \lambda^{2}}{(4 \pi)^{2} d^{2} L}
$$

where, $P_{t}$ is the signal power of transmitter; $G_{t}$ is the transmit antenna gain; $G_{r}$ represents receive antenna gain; $L$ is independent system for the propagation loss factor; $\lambda$ is the signal wavelength; $d$ denotes the distance between receiving terminal and transmitting terminal. If set $G_{t}=G_{r}=L=1$, a reference distance $d_{0}$ is defined as $1 \mathrm{~m}$, and the received power can be expressed as follow:

$$
\begin{aligned}
P_{r}(d) & =\frac{P_{t} G_{t} G_{r} \lambda^{2}}{(4 \pi)^{2} d^{2} L}=\frac{P_{t} G_{t} G_{r} \lambda^{2}}{(4 \pi)^{2} d_{0}^{2} L} \cdot\left(\frac{d_{0}}{d}\right)^{2} \\
& =P_{r}\left(d_{0}\right) \cdot\left(\frac{d_{0}}{d}\right)^{2}
\end{aligned}
$$

where, $P_{r}\left(d_{0}\right)$ is received signal power which is measured at $d_{0}$ and it's also a practical testing parameter.

In practical circumstances, due to the presence of a number of tracks, noise, etc., the received power have great different with the free-space propagation model calculation results. Studies have shown that long-distance channel fading characteristics lognormal distribution, the common logarithm of the distance path loss model is

$$
P_{L}(d)=P_{L}\left(d_{0}\right)+10 n \lg \left(d / d_{0}\right)+N(\sigma)
$$

where, $n$ is the path loss factor which is related to the environment and it generally ranges from 2 to 4 ; $N$ is Gaussian random variable and its mean value is 0 and standard deviation $\sigma$ ranges from 4 to 10 .

RSSI of the receiving node is:

$$
R S S I=P_{t}-P_{L}(d)
$$

where, $P_{t}(\mathrm{dBm})$ is the signal power of the transmitter; signal strength at the reference distance $d_{0}(1 \mathrm{~m})$ is $\mathrm{A}$, then

$$
R S S I=A-10 n \lg (d)-N(\sigma)
$$

According Eq. (5) we can calculate the distance $d$.

\section{Localization Model}

Assume that there are $m$ anchor nodes and $n$ unknown nodes in WSNs. The vector $S=\left[z_{1}, z_{2}, \cdots, z_{m+n}\right]$ represents the initial position of the sensor nodes, where $z_{i}=\left[x_{i}, y_{i}\right]^{T} . m$ anchor nodes located $\left(x_{1}, y_{1}\right),\left(x_{2}, y_{2}\right), \cdots,\left(x_{m}, y_{m}\right)$. So the location problem becomes how to obtain the location of unknown nodes $S=\left[S_{x}, S_{y}\right]$ according to the location of anchor nodes, where $S_{x}=\left[x_{m+1}, x_{m+2}, \cdots, x_{m+n}\right], \quad S_{y}=\left[y_{m+1}, y_{m+2}, \cdots, y_{m+n}\right]$.

Suppose the coordinates of $i$ th anchor node are $\left(x_{i}, y_{i}\right)$, the coordinates of the unknown nodes are $\left(x_{0}, y_{0}\right)$, and the distance between the unknown node and the $i$ th anchor node is $\sqrt{\left(x_{i}-x_{0}\right)^{2}+\left(y_{i}-y_{0}\right)^{2}}$, where $i$ is, respectively, 1 , $2,3, \ldots, \mathrm{m}$. Because of the distance error, the position $\left(x_{0}, y_{0}\right)$ called estimated position is not its real position. In order to let the estimated position and the real position to be closer, the error was defined as follow:

$$
e_{i}=d_{i}-\sqrt{\left(x_{i}-x_{0}\right)^{2}+\left(y_{i}-y_{0}\right)^{2}}
$$

where, $d_{i}$ is the measured distance according to RSSI. 


\section{A Localization Algorithm Based on Particle Swarm Optimization and Quasi-Newton Algorithm for Wireless Sensor Networks}

\subsection{PSO algorithm}

PSO is an evolutionary metaheuristic method proposed by Kennedy and Eberhart [7]. In PSO, each individual is called a particle, representing a potential solution. A swarm consists of $\mathrm{m}$ particles. The position of the $i$ th particle at the $k$ th iteration is used to evaluate the particle and represent the candidate solution for the optimization problem. During the search process, each particle can estimate its own fitness value of position and remember the best position visited by itself which called the local optimal value p best; also it is must remember the best position of all particles by now which is called global optimal value g best.

The new velocity and position of particle $i$ at next iteration can be calculated according to the Eqs. (7) and (8):

$$
\begin{gathered}
V_{i}^{k+1}=\omega V_{i}^{k}+c_{1} r_{1}\left(p_{i}^{k}-z_{i}^{k}\right)+c_{2} r_{2}\left(p_{g}^{k}-z_{i}^{k}\right) \\
z_{i}^{k+1}=z_{i}^{k}+V_{i}^{k+1}
\end{gathered}
$$

where, $i$ is respectively $1,2, \ldots, \mathrm{m}, k$ denotes iteration number , $r_{1}$ and $r_{2}$ are random numbers between [0,1], and $c_{1}, c_{2}$ are respectively the cognitive and social learning parameters between [0,2], $\omega$ is the inertia weight, which provides a balance between global and local explorations.

The bigger value of $\omega$ is beneficial for particles to jump out of local minimum points, while the smaller value of $\omega$ is favor the algorithm convergence. For global exploration, usually using a big initial inertia weight to obtain appropriate seed, and using the small inertia weight in the late algorithm to improve the local search ability and speed up convergence. As originally developed, $\omega$ often decreases linearly with the number of iterations. So $\omega$ can be set according to the following equation [8-10]:

$$
\omega=\omega_{\max }-\left(\omega_{\max }-\omega_{\min }\right) k / k_{\text {max }}
$$

where, $\omega_{\max }, \omega_{\min }$ is initial and final inertia weight respectively, $k_{\max }$ is the maximum number of iterations, and $k$ is the current number of iterations. This algorithm is called LWPSO (linearly decreasing weight particle swarm optimization). A number of experiments show that LWPSO can obtain best results when $w$ is decreasing from 0.9 to 0.4 .

According to Eq. (6), suppose there are $n$ neighbor anchor nodes nearing unknown node $i$, so the error equation can be expressed as follow:

$$
\sum_{\mathrm{i}=1}^{\mathrm{n}} e_{\mathrm{i}}=\sum_{\mathrm{i}=1}^{\mathrm{n}}\left(d_{i}-\sqrt{\left(x_{i}-x_{0}\right)^{2}+\left(y_{i}-y_{0}\right)^{2}}\right)^{2}
$$

In order to reduce the cumulative localization error, the error equation can be defined as follow:

$$
\sum_{\mathrm{i}=1}^{\mathrm{n}} e_{\mathrm{i}}=\sum_{\mathrm{i}=1}^{\mathrm{n}} \frac{\left(d_{i}-\sqrt{\left(x_{i}-x_{0}\right)^{2}+\left(y_{i}-y_{0}\right)^{2}}\right)^{2}}{d_{i}}
$$

Eq. (11) is the fitness function of PSO.

In the search process of PSO, each particle calculates the minimum fitness value according to coordinates of anchor nodes and the measured distance, and then gets the local optimum value $\mathrm{p}$ best and the global optimum value g best in an iterative process. Iterative search process repeated until reaching the convergence point. To avoid falling into local optimum, all the particles iterate 30 times and stop PSO algorithm.

In the localization algorithm, when an unknown node collects coordination of three or more anchor nodes, it can calculate its own coordination through PSO. Estimating coordinates of unknown nodes can be seen as an optimization problem which is the minimum fitness function values.

\subsection{Quasi-Newton methods}

Quasi-Newton method is an efficient method for solving optimization problems, the most popular Quasi-Newton algorithms is the BFGS method (named for its discoverers Broyden, Fletcher, Goldfarb and Shanno).The BFGS algorithm is an iterative method for solving nonlinear optimization problems. 
Quasi-Newton algorithm overcomes the shortcomings of Newton algorithm which needing derivation and inversion, and uses the approximate Hessian $B_{k}$ in place of the true Hessian, which simplify the computing process, but maintain the super linear rate of convergence [11]. Using BFGS to solve a nonlinear optimization needs an initial value, which has a direct impact on results and convergence [12].

BFGS algorithm takes the approximate value which obtains by PSO as initial value to improve the local searching ability. Because BFGS is a direct calculation method, it can improve the localization precision and speed significantly. And it also can effectively reduce the possibility of the result falling into local optimum according the initial value by the PSO.

\subsection{PSO and Quasi-Newton iterative algorithm}

A hybrid algorithm which called PSO-QN based on PSO and Quasi-Newton iterative algorithm was put forward, which selects initial location by PSO and gets final result through Quasi-Newton iterative algorithm, Fig. 1 is the flow chart of the hybrid algorithm and its steps can be expressed as follow:

(a) Initialize particle swarm including the number of particles, the positions and velocities of the particles;

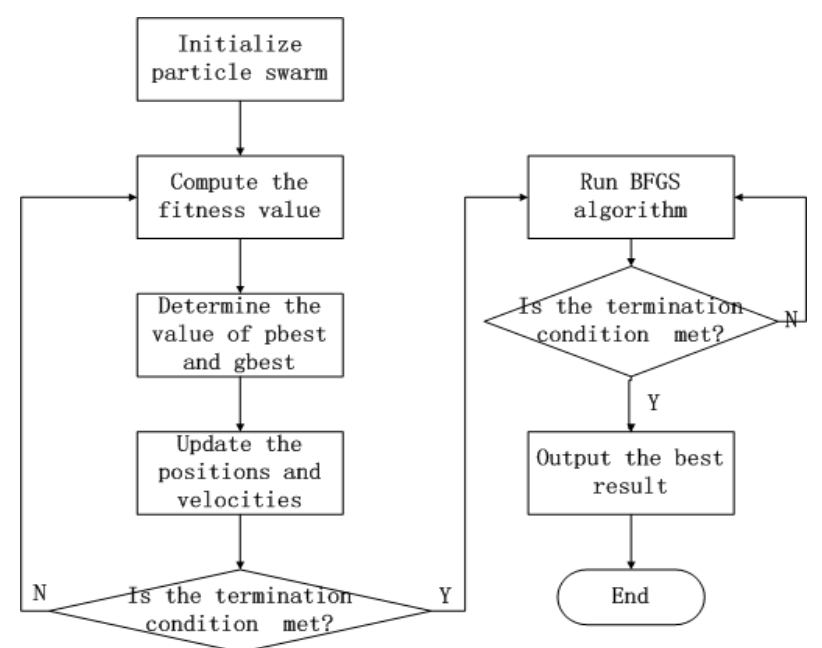

Fig. 1 The flow chart of the hybrid algorithm. (b) Compute the fitness value of each particle, and store current position and fitness value in each particle as p best, and then choose the best position and fitness value as the current global solution g best;

(c) Update the particle's velocity and position according to the Eqs. (7) and (8);

(d) Compare particle's fitness evaluation with its $\mathrm{p}$ best. If current value is better than p best, then set the current value as p best;

(e) Compare all of current p best with g best, and select the best as g best;

(f) If the termination condition (the maximum number of iterations or a sufficiently good fitness) is met, return the current global best solution $P g$, and jump to step $g$ ); otherwise, $k=k+1$, and update the inertia weight, go to step b);

(g) Run Quasi-Newton algorithm. Use the current global best particle $P g$ which got in step f) as initial value to iterate;

(h) If the termination condition is met, then output the current result as the problem optimal solution, otherwise use current result as the initial value of Quasi-Newton algorithm to iterate again.

\section{Simulation and Analysis}

To test and verify the performance of the localization algorithm, the simulation uses the Matlab to evaluate and compare the performance of our algorithm to LWPSO. There are 100 sensor nodes including a number of unknown nodes and some anchor nodes, which are randomly distributed in the $100 \mathrm{~m} \times 100 \mathrm{~m}$ square area. Fig. 2 is nodes distribution in a particular simulation. Fig. 3 is the location results. The algorithms were compared with the LWPSO algorithm, the number of particle is 20 , the maximum number of iterations is 50, inertia weight $\omega_{\max }=0.9, \omega_{\min }=0.2, c_{1}=2, c_{2}=2$, the communication radius among nodes is $R$.

(1) The number of anchor nodes and localization error

The density of anchor nodes reflects its distribution 


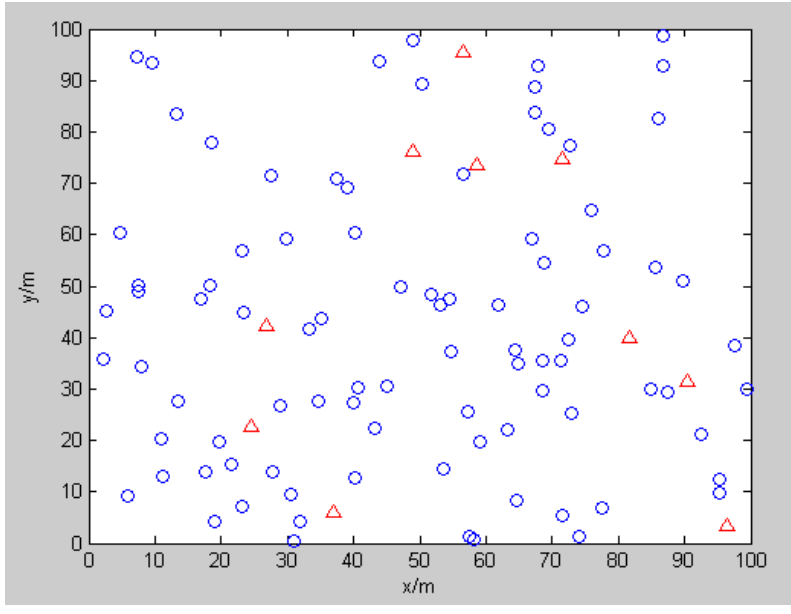

Fig. 2 Nodes distribution.

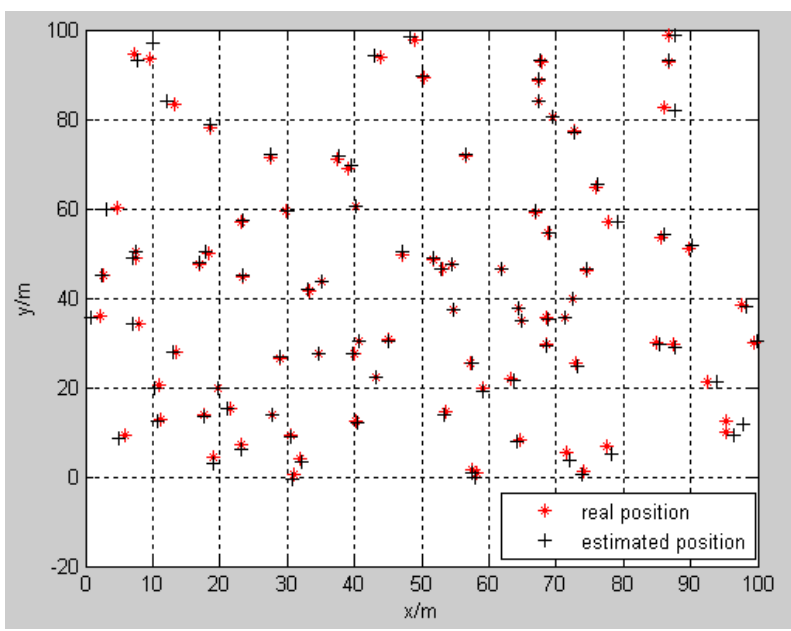

Fig. 3 The location result.

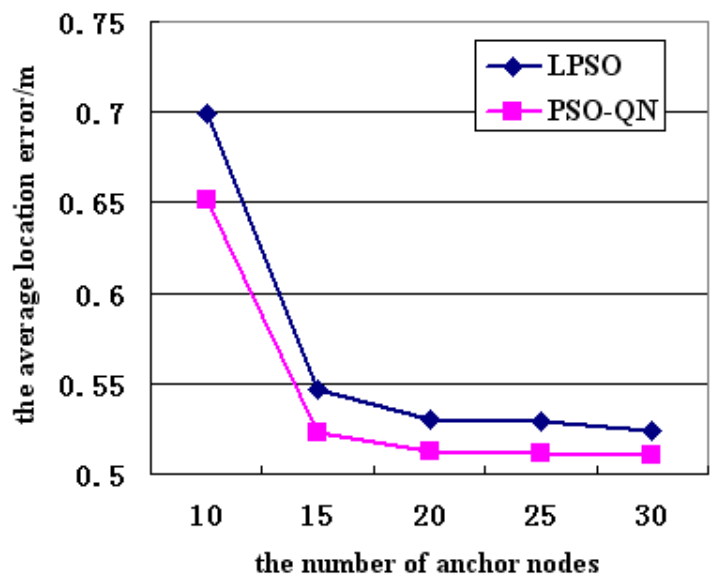

Fig. 4 The number of anchor nodes and average localization error.

in the whole area, due to the high cost of the anchor nodes, it's usually want to use less anchor nodes to locate unknown nodes as many as possible. Fig. 4 shows the number of anchor nodes and average localization error, and the localization accuracy is proportional to the number of anchor nodes, but the average localization error became relative stable when the number of anchor nodes is up to certain degree. The PSO-QN algorithm has smaller average localization error than LWPSO algorithm in the same condition.

(2) The communication radius and localization error

Fig. 5 shows the relation between the average localization error and the communication radius under the condition of the number of anchor nodes is $10 \%$. As the increase of communication radius, the average localization error decreased, mainly due to the increase of communication radius, the number of anchor nodes nearing the unknown nodes and the signal strength increase, and so the ranging will be more accurate. The PSO-QN algorithm has smaller average location error than LWPSO.

(3) Ranging error and localization error

Because the distance measured from RSSI is different in vary condition, which could get different ranging error. Ranging errors directly affect the accuracy of measurements distance between nodes, and then affects the localization accuracy. Fig. 6 shows the average localization error based on different ranging error, which the proportion of anchor nodes in (a), (b) and (c) is $10 \%, 15 \%$ and $30 \%$ respectively. The

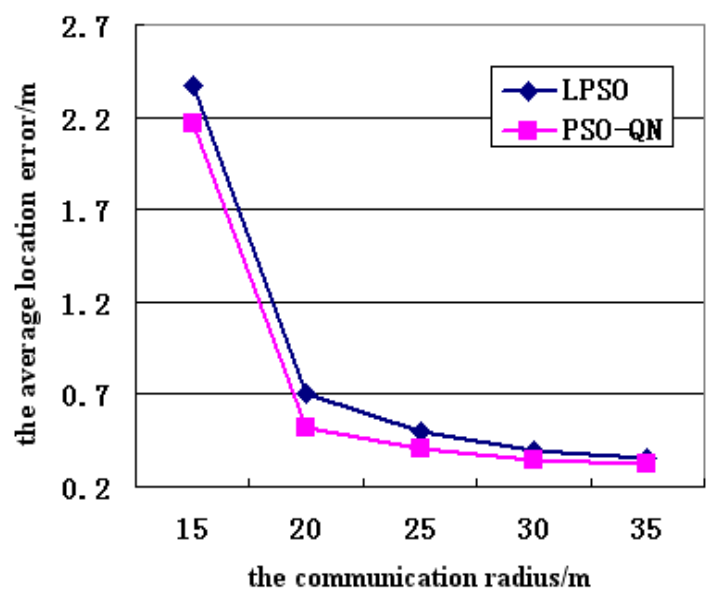

Fig. 5 The relation between the average localization error and the communication radius. 


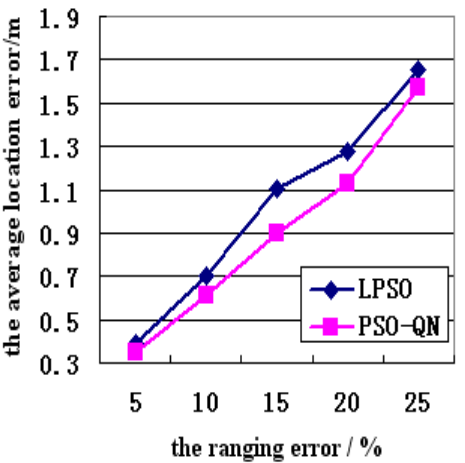

(a)

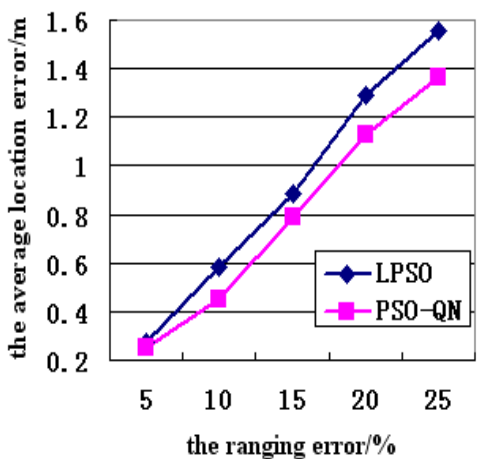

(b)

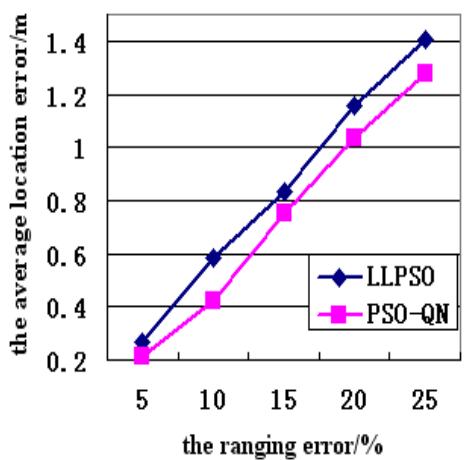

(c)

Fig. 6 The relation between the average localization error and the ranging error.

average localization error increases with the increasing of ranging error, but the average location error of the PSO-QN algorithm is lower than LWPSO. Especially, when the ranging error is big, the localization accuracy of the algorithm is still high.

\section{Conclusions}

In order to improve the localization accuracy in WSNs, this paper discusses the localization method based on improved particle swarm optimization algorithm and Quasi-Newton algorithm, which obtains the approximate solutions through PSO, and then makes local search and gets the final results by BFGS. The simulation shows that the algorithm can effectively improve the localization accuracy.

\section{References}

[1] Akyildiz, I. F., Su, W., and Sankarasubramaniam, Y. 2002. "Wireless Sensor Network: A Survey." Computer Networks 38 (4): 393-422.

[2] He, T., Huang, C. D., and Blum, B. M. 2003. "Range-Free Localization Schemes in Large Scale Sensor Networks." In Proceedings of the Ninth Annual International Conference on Mobile Computing and Networking, 81-95.

[3] Aboelaze, M., and Aloul, F. 2005. "Current and Future Trends in Sensor Networks: A Survey.” In Proceedings of Second IFIP International Conference on Wireless and Optical Communications Network, 551-55.
[4] Rudafshani, M., and Datta, S. 2007. Localization in Wireless Sensor Networks.” In Proceedings of Information Processing in Sensor Networks, 51-60.

[5] Luthy, K. A., Grant, D. E., and Henderson, T. C. 2007. "Leveraging RSSI for Robotic Repair of Disconnected Wireless Sensor Networks.” In Proceedings of 2007 IEEE International Conference on Robotics and Automation, 10-14.

[6] Girod, L., Bychovskiy, V., Elson, J., and Estrin, D. 2002. "Locating Tiny Sensors in Time and Space: A Case Study." In Proceedings of the 2002 IEEE International Conference on Computer Design, IEEE Press, 214-19.

[7] Kennedy, J., and Eberhart, R. C. 1995. "Particle Swarm Optimization.” In Proceedings of IEEE International Conference on Neural Networks, 1942-48.

[8] Shi, Y., and Eberhart, R. 1999. "Empirical Study of Particle Swarm Optimization.” In Proceeding of the 1999 Congress of Evolutionary Computation, Los Alamitos: IEEE Press, 1945-50.

[9] Poli, R., Kennedy, J., and Blackwell, T. 2007. "Particle Swarm Optimization: An Overview.” Swarm Intell 1: 33-57.

[10] Khairy, M., Fayek, M. B., and Hemayed, E. E. 2011. "PSO2: Particle Swarm Optimization with PSO-Based Local Search.” Presented at the IEEE Congress of Evolutionary Computation (CEC), NewOrleans, LA, United states.

[11] Yuan, G., and Lu, X. 2011. "An Active Set Limited Memory BFGS Algorithm for Bound Constrained Optimization.” Applied Mathematical Modeling 35 (7): 3561-73.

[12] Yang, W. A., Hu, C., and Li, M. 2010. “A New Tracking System for Three Magnetic Objectives.” IEEE Transactions on Magnetics 40 (12): 4023-29. 